\title{
DU MAINTIEN DES APPELS A LA COUR SUPREME DU CANADA EN MATIERE DE DROIT CIVIL
}

\author{
GÉRALD A. BEAUDOIN*
}

L'auteur de cet article analyse la question du maintien des appels en droit civil à la Cour Suprême. Dans le contexte fédéral canadien, il se déclare favorable au maintien de ces appels. Il y trouve plus d’avantages que de désavantages. Le Canada ayant deux systemes de droit privé, il convient que le plus haut tribunal du pays se prononce en ces matières il s'agit d'une richesse pour notre pays. Il y a moyen d'assurer la pureté de l'un et de l'autre système.

The author of this article analyses the question of the retention of the civil law appeals to the Supreme Court of Canada. He is in favour of such appeals in our federation. We have in Canada two private law systemss one inspired from the Common Law of England and the other from "le droit civil" of France. It is appropriate for our highest tribunal to render judgments in both systems. This is a great advantage for our country. Steps may be taken to assure the purity of both systems.

G. A.B.

Le plus haut tribunal canadien devrait-il continuer à entendre et à trancher les appels en droit civil provenant du Québec?

Pour situer le problème dans une juste perspective, il importe de replacer la Cour Suprême dans le cadre constitutionnel canadien.

Contrairement à la Cour Suprême des Etats-Unis dont l'existence est prévue par la loi fondamentale du pays, ${ }^{1}$ notre Cour Suprême doit sa création à une simple loi fédérale passée sous l'autorité de l'article 101 de l'Acte de l'Amérique du Nord britannique de 1867, huit ans après l'avènement du régime fédératif au Canada. ${ }^{2}$ Notre plus haute Cour n'a pas à proprement parler de statut constitutionnel puisque son existence, sa composition, et, son fonctionnement ne sont pas prévus dans la loi fondamentale du pays. En juin 1971, à Victoria, les autorités fédérale et provinciales se sont mises d'accord pour prévoir dans un texte à portée constitutionnelle l'existence et la composition de notre Cour Suprême ainsi que le mode de nomination de ses juges. ${ }^{3}$ Mais la Charte de Victoria n'a pas vu le jour, comme on le sait à la suite du refus opposé par le Québec. La Cour Suprême n'existe qu'en vertu d'une loi que le Parlement central est libre de modifier, voire même d'abroger.

L'article 101 de la Constitution autorise le Parlement central à ériger une cour générale d'appel pour tout le pays. Mais, en 1875, le Comité Judiciaire du Conseil Privé continuait de "chapeauter" le pouvoir judiciaire canadien et notre Cour Suprême ne fut réellement suprême qu'en décembre 1949 lorsque les derniers appels au Conseil Privé furent abolis. ${ }^{4}$

Aussi, quand nous disons qu'en cette année 1975 nous célébrons le centenaire de la Cour Suprême, ceci n'est exact que sur le plan de sa création. Cette cour n'est suprême que depuis un quart de siècle.

Selon la jurisprudence, ${ }^{5}$ le Parlement fédérale détient seul le pouvoir de déterminer la juridiction d'appel de la Cour Suprême. Ce Parlement a reconnu

- Dean, Université D'Ottawa, Faculté De Droit.

1 Article III, section I, de la Constitution des Etats-Unis de 1787.

2 Acte pour établir une Cour suprême et une Cour d'Echiquier pour le Canada, S.C. 1875 , c. 11 (sanctionnée le 8 avril 1875).

${ }^{3}$ Articles 22 à 42 de la Charte constitutionnelle canadienne. (Charte de Victoria).

1 Loi modifiant la loi de la Cour suprème, S.C. 1949, c. 37 (sanctionnée le 10 décembre 1949).

- Crown Grain Co. v. Day, [1908] A.C. 504. 
à la Cour Suprême un droit d'appel dans les matières non seulement relatives au droit fédérale mais également dans plusieurs domaines du droit provincial. De plus, la Cour Suprême détient un "Judicial Review" en matière constitutionelle de sorte que comme aux Etats-Unis, elle est la gardienne de la Constitution et constitue la clef de voûte du pouvoir judiciaire.

Nous n'avons pas au Canada comme dans certains autres pays une cour constitutionnelle. C'est notre Cour Suprême qui en dernier ressort départage les autorités fédérale et provinciales en matière de conflits constitutionnels. C'est également le cas aux Etats-Unis. On a suggéré en certains milieux l'établissement d'une cour constitutionnelle. En 1968, le Québec en a fait la recommandation lors de la Conférence constitutionnelle.

Il y a évidemment beaucoup de mérite à la création d'une telle cour constitutionnelle mais elle comporte également de sérieux désavantages. Aussi, même si ce sujet ne fait pas directement l'objet de la présente étude, je puis dire ici que je ne favorise pas l'établissement d'un tel tribunal. La Cour Suprême doit jouer le rôle de cour constitutionnelle. Une cour de justice de dernier ressort est moins sujette à l'arbitrage politique qu'une cour constitutionnelle dont les membres seraient nommés par les chambres législatives ou encore de concert par les autorités fédérale et provinciales. ${ }^{\circ}$ C'est le système qu'ont adopté les auteurs de la fédération américaine. Au surplus, les causes constitutionnelles ne sont pas nombreuses au point d'exiger un tribunal spécial. Enfin, une cour constitutionnelle n'écarterait pas la nécessité pour les pouvoirs provinciaux et fédéral de se rencontrer et de négocier sur le plan constitutionnel. Ceci fait partie de notre histoire constitutionnelle.

L'indépendance des juges de la Cour Suprême n'existe qu'en vertu d'une tradition quoique solidement ancrée et d'une simple disposition d'une loi fédérale. ${ }^{7} \mathrm{Au}$ niveau des cours supérieures l'article 99 de la Constitution assure l'indépendance judiciaire. Aucun juge ne peut être démis de ses fonctions sauf à la suite d'une adresse des deux chambres fédérales. Une telle destitution est inconnue dans notre histoire. ${ }^{8}$

Le caractère bilingue de la Cour Suprême n'est pas assuré par la Constitution, si ce n'est de façon assez éloignée par l'article 133 de la Constitution qui prévoit inter alia que les plaidoiries orales et écrites devant cette cour peuvent se faire en français ou en anglais. Mais depuis 1969, la loi fédérale sur les langues officielles ${ }^{9}$ prévoit que les jugements de la Cour doivent être rendus dans les deux langues.

Au Canada, il existe deux systèmes juridiques: l'un de droit civil qui s'applique au Québec et l'autre de Common Law qui est en vigueur dans les neuf autres provinces. L'Acte de Québec de $1774^{10}$ autorisait les Québécois à vivre sous un régime de droit privé d'inspiration français. Avant la Confédération, Sir Georges Etienne Cartier avait fait codifier les lois civiles du Bas-Canada ${ }^{11}$ et le code civil est venu en vigueur en août $1866,{ }^{12}$ onze mois avant l'avènement de la Confédération.

- Voir G. A. Beaudoin, La Cour suprême du Canada et la constitutionnalité des Lois, dans Actualité du contrôle juridictionnel des lois, à 187.

7 Supra, n. 2, art. 9.

8 Voir G. A. Beaudoin, Le système judiciaire canadien, (1968) 28 R. du B. 99.

${ }^{\circ}$ Loi sur les langues officielles, S.C. $1968-69$, c. 54.

1014 Geo. III, c. 83 (Imp.). Voir aussi Chapais, Cours d'Histoire du Canada, tome 1, par J. P. Garneau (éd.), à 325-333.

11 Voir la préface du Petit Code civil annoté de la Province du Québec, par L.-J. De La Durantaye.

12 Id. 
Maintenant que la Cour Suprême a été située dans son décor, on peut se demander s'il serait préférable que les appels en droit civil cessent au niveau de la Cour d'Appel du Québec? Le droit privé étant de compétence provinciale, ${ }^{18}$ pourquoi faut-il que la Cour Suprême se prononce en cette matière? Ne devraitelle pas se restreindre au droit "fédéral" et au droit constitutionnel? Des juristes éminents ont déjà proposé que les appels en droit civil soient entendus en dernier ressort par notre Cour d'Appel, ${ }^{14}$ afin de bien préserver le génie du droit civil.

Je suis favorable au maintien des appels en matière civile à la Cour Suprême. Notre pays constitue un modèle vivant de droit compare. ${ }^{15}$ Comme nous avons deux systèmes de droit au Canada, les appels en matière civile et en Common Law devraient être tranchés par le tribunal suprême. Il s'agit là d'une richesse dont nous aurions tort de ne pas tirer profit. Cependant pour ne pas trop alourdir le fardeau de la Cour, cette dernière ne devrait se prononcer en droit civil et en common law que dans des matières de grande importance.

On objecte que comme les deux tiers des juges à la Cour Suprême ne sont pas de formation civiliste le génie du droit civil peut être en danger. En effet, cinq juges de la Cour d'Appel du Québec se prononcent en droit civil et au niveau de la Cour Suprême trois juges civilistes avec deux collègues de common law peuvent renverser cette décision, voire même un seul juge civiliste avec deux juristes de common law et deux civilistes dissidents peuvent infirmer le jugement des cinq juges civilistes de la Cour d'Appel.

Si les appels en droit civil sont maintenus à la Cour Suprême, peut-on garantir la pureté du droit ciyil?

Certains auteurs ont écrit que les chambres étanches et spécialisées au sein de la Cour Suprême n'étaient pas la solution désirée, car il est difficile d'isoler le droit civil des autres problèmes de droit. ${ }^{16}$

Certains juristes prônent au contraire la création d'une chambre civile.

Pourrait-on mieux assurer la pureté du droit civil du Québec en augmentant le nombre des juges? J'ai déjà suggéré que le nombre des juges soit porté à quinze. ${ }^{17}$ On garderait alors la même proportion entre les juges québécois et les juges de common law, soit un tiers/deux tiers, ce qui voudrait dire que cinq juges seraient du Québec et dix des autres provinces: par exemple cinq juges de l'Ontario trois juges des provinces de l'Ouest et deux juges des provinces Maritimes. Un pareil système aurait le mérite de créer un banc de droit civil de cinq juges à la Cour Suprême et d'établir une parité avec la Cour d'Appel. L'augmentation du nombre des juges ne peut se justifier que parce que nous avons deux systèmes de droit. Si nous n'en avions qu'un, comme c'est le cas aux Etats-Unis, neuf juges suffiraient évidement.

Cette proposition retient encore mon attention; elle comporte des avantages évidents. Elle répondrait fort adéquatement à toutes les critiques formulées depuis 1875. Elle soulève cependant d'autres difficultés. Certains prétendent qu'il serait inefficace de hausser le nombre actuel des juges. L'efficacité deviendrait alors inversement proportionnelle au nombre de juges nommés. Il se

18 British North America Act, 1867, 30 \& 31 Vict., c. 3, art. 92 (13) (Imp.).

14 G. A. Beaudoin, supra, n. 8.

${ }^{15}$ L'expression je crois vient du regretté Professeur Louis Baudouin.

16 Voir G. E. Ledain, Concerning the Proposed Constitutional and Civil Law Specialization at the Supreme Court Level, (1967) Thémis 107.

17 Voir G. A. Beaudoin, La révision constitutionelle et le statut de la Cour suprême, (1970) R.G.D. 257. 
pourrait que l'objection soit bien fondée encore qu'elle ne paraisse pas évidente. Des études plus approfondies devraient être entreprises avant que l'on s'engage sur cette voie. Mais encore une fois cette suggestion n'est pas à écarter.

Sauf au pénal, la Cour Suprême dorénavant n'entendra les appels que sur permission. ${ }^{18}$ Cette réforme va alléger le fardeau de la Cour Suprême et écarter les appels qui n'impliquent que des sommes d'argent et qui ne soulèvent pas de graves problèmes juridiques. Les appels à la Cour Suprême étant restreints aux matières les plus importantes, il est peut-être possible pour les neuf juges d'entendre sans trop de peine tous les appels en droit civil et en common law qui ont une grande signification pour le pays tout entier.

Cependant là encore un banc de juristes civilistes pourra renverser un banc de civilistes de la Cour d'Appel qui comprend un plus grand nombre de juges. A moins d'augmenter le nombre des juges, on ne peut éviter cette éventualité.

Disons en passant que la Législature québécoise peut toujours corriger pour l'avenir les affets d'un jugement de la Cour Suprême en droit civil qu'elle trouve peu respectueux du génie du droit civil. Mais il s'agit évidement là d'un contrôle ex post facto et non entièrement satisfaisant.

Certains se sont demandés si l'on n'a pas exagéré les différences entre les principes du droit civil et ceux de la Common Law. Les résultats ne sont-ils pas souvent les mêmes. Peut-êtrel Cependant, la technique et l'approche différent substantiellement et il faut faire preuve de grande prudence pour ne pas gêner le génie d'un système particulier.

Enfin le maintien des appels en droit civil à la Cour Suprême soulève la question de la nomination des juges. Aux termes de la loi sur la Cour Suprême, les juges sont nommés par l'exécutif fédéral. ${ }^{10}$ Il n'y a aucune participation des provinces. Comme la Cour Suprême est appelée à trancher des litiges constitutionels entre les provinces et le pouvoir fédéral et à se prononcer dans des matières provinciales, plusieurs juristes et politicologues ont suggéré que les provinces participent au processus de nomination des juges. A la Conférence constitutionnelle de Victoria l'autorité fédérale elle-même a convenu du bienfondé de la thèse voulant que les provinces soient appelées à participer à la nomination des juges. ${ }^{20}$

Quelle forme devrait prendre cette participation? La Charte de Victoria de 1971 proposait que le Procureur Général de la province concernée soit appelé à donner son accord. Le procureur général du Canada et celui de la province concernée doivent s'entendre dans la mesure du possible. A défaut, un collège est mis sur pied avec pouvoir de recommandation. Actuellement, la prérogative de nomination relève de l'autorité centrale. Par voie d'amendement constitutionnel on pourrait prévoir un mécanisme d'intervention de la part des provinces qui s'inspirerait de la Charte de Victoria. Le concours des provinces devrait être institutionnalisé au niveau de la nomination des juges de la Cour Suprême.

Les juges des Cours d'Appel et de la Cour Supérieure sont également nommés par le Gouvernement central ${ }^{21}$ même s'il s'agit de cours provinciales. Mais sur ce plan la critique est moins vive car il ne s'agit pas de cours de dernier ressort.

18 Loi modifiant la loi sur la Cour suprême et modifiant en conséquence la loi sur la Cour fédérale, S.C. 1975 , c. 18 , art. 5 .

19 Supra, n. 2, art. 4.

20 Voir les articles 26 a 32 de la Charte de Victoria. 
Aux Etats-Unis le Président nomme les juges mais le Sénat qui représente les Etats concourt à leur nomination. ${ }^{22}$ Au Canada, le Sénat ne saurait jouer ce rôle. Il faut donc faire intervenir les provinces. Une telle participation se justifie car la Cour est arbitre en droit constitutionnel; si la Cour doit continuer à entendre les appels en droit civil et en common law, c'est-d̀-dire des appels dans le domaine où les provinces ont compétence exclusive, il existe une raison de plus pour faire participer les provinces à la nomination des juges.

Il m'apparaît que si les provinces étaient appelées à participer à la nomination des juges de la Cour Suprême, et si le nombre des juges était porté à quinze, il n'existerait plus aucune raison de vouloir faire cesser les appels en droit civil au niveau de la Cour d'Appel du Québec. Je serai encore favorable au maintien desdits appels, même si le nombre des juges demeurait à neuf, pourvu que la première condition au moins se réalise.

21 Supra, n. 13, art. 96.

22 Article II, section II, alinéa 2 de la Constitution américaine. 